\title{
Environmental and energy assessment of the use of Andesite in cement
}

\section{Evaluation environnementale et énergétique de l'utilisation de l'Andésite dans le ciment}

\author{
Moussa HAMIDI ${ }^{1}$, Larbi KACIMI ${ }^{2}$, Pierre CLASTRES ${ }^{3}$ \\ ${ }^{1}$ Université de Djilali Bounaama,, Faculté des sciences et de la technologie, Khemis Miliana W. Ain Defla, Algérie \\ ${ }^{2}$ Université USTO, Faculté de Chimie, Oran, Algérie. \\ ${ }^{3}$ Université de Toulouse, Laboratoire LMDC, INSA-UPS, Toulouse, France.
}

\begin{abstract}
The present study is a contribution to the research of new sources of supply of additions and raw materials in order to valorize local materials in the manufacture of cement. It consists in checking the environmental validity of the industrial use of andesite by evaluating the energy consumption of manufacturing andesitic cement. This evaluation was made on CEMI 52.5R type cement mortars whose basic constituent is clinker, with substitution rates of raw and calcined andesitic rock at $800{ }^{\circ} \mathrm{C}$ varying from $0-40 \%$. and this through mechanical performance tests at several deadlines ( 2 days, 7 days, 28 days and 90 days). The results show that the analysis of the influence on the environment of this addition, in its raw state or calcined at $800^{\circ}$ $\mathrm{C}$, made it possible to say that globally the impact of andesite on the matrices seems positive to long term. The replacement of the cement by andesite systematically induces a reduction in the energy required to produce one ton of binder, due to the lower energy demand for grinding than that required for the thermal treatment of the clinker. Overall, the energy cost of andesite cement (in kWh per MPa compressive strength) is lower than that of ordinary cement for low rates of clinker replacement and obtaining cements with moderate strengths. Keywords: Andesite; Cement; Impact; Environment; Energy.
\end{abstract}

\begin{abstract}
Résumé. La présente étude est une contribution à la recherche de nouvelles sources d'approvisionnement en ajouts et en matières premières en vue de valoriser des matériaux naturels locaux dans la fabrication du ciment. Elle consiste à vérifier la validité environnementale de l'utilisation industrielle de l'andésite en évaluant la consommation énergétique de fabrication du ciment andésitique. Cette évaluation a été faite sur des mortiers de ciment de type CEMI 52.5R dont le constituant de base est le clinker, avec des taux de substitution de roche andésitique brute ou calcinée à $800^{\circ} \mathrm{C}$ variant de $0-40 \%$, et ce par le biais de tests de performances mécaniques à plusieurs échéances ( 2 jours, 7 jours, 28 jours et 90 jours). Les résultats montrent que l'analyse de l'influence sur l'environnement de cette addition, à l'état brut ou calcinée à $800^{\circ} \mathrm{C}$, a permis de dire que globalement l'impact de l'andésite sur les matrices semble positif à long terme. Le remplacement du ciment par l'andésite induit systématiquement une diminution de l'énergie nécessaire pour produire une tonne de liant, en raison de la demande d'énergie de broyage plus faible que celle nécessaire au traitement thermique du clinker. Globalement, le coût énergétique du ciment d'andésite (en kWh par MPa de résistance à la compression) est inférieur à celui du ciment ordinaire pour les faibles taux de remplacement de clinker et l'obtention de ciments à résistances modérées.
\end{abstract}

Mots-Clés : Andésite ; Ciment ; Impact ; Environnement ; Energie.

\section{Introduction}

La recherche de nouvelles sources d'approvisionnement en ajouts et en matières premières en vue de valoriser des matériaux naturels locaux dans la fabrication du ciment, est devenue une préoccupation écologique majeure pour les cimentiers, en termes de préservation de l'environnement et développement durable. Pour limiter l'impact environnemental relatif au procédé de production du ciment, l'emploi de ciment partiellement substitué par des additions minérales est envisagé comme liant pour l'industrie cimentaire. Cette substitution fait actuellement partie des développements les plus récents dans la production du ciment, en apportant une amélioration sur les propriétés mécaniques des matériaux cimentaires et en réduisant la consommation du clinker, en contribuant de manière simple et économique à résoudre les problèmes liés à 
l'environnement. La présente étude consiste à vérifier la validité environnementale de l'utilisation industrielle de l'andésite sur l'environnement et ce en évaluant la consommation énergétique de fabrication du ciment à base de roche andésitique. Les andésites sont des dépôts volcaniques riches en silice (53-63\%) et de composition chimique et minéralogique variable [1-2]. Les propriétés physiques et mécaniques de l'andésite sont généralement satisfaisantes aux exigences acceptables pour leur utilisation dans divers domaines [3-5]. Ces propriétés lui permettent notamment la possibilité d'être utilisée comme matière première pour la fabrication du verre [6-12]. Les travaux antérieurs sur l'andésite Algérienne, ont montré qu'il s'agit d'andésites appartenant à la série calcoalcaline, à texture microlitique fortement porphyrique. L'évaluation des impacts environnementaux liés à l'utilisation de cette roche dans la fabrication du ciment a été faite sur des mortiers à ciment de type CEMI 52.5R et d'un mortier dont le constituant de base est le clinker, avec des taux de substitution de roche andésitique brute et calcinée à $800^{\circ} \mathrm{C}$ variant de $0-40 \%$, et ce par le biais des tests de performances mécaniques à plusieurs échéances (2, 7, 28 et 90 jours). L'andésite utilisée est de provenance de la région de la wilaya de Tipaza-Algériee.

\section{Expérimentation}

\subsection{Matériaux utilisés}

Les matériaux utilisés dans la formulation des mortiers et des pâtes pour tous les modèles expérimentaux sont: le ciment, le clinker, le gypse, l'andésite, le sable et l'eau. Selon la norme européenne [EN 197-1] [15], trois types de ciment, nommés A, B et C ont été utilisés:

- Ciment A : CEM I 52,5R, fabriqué à l'usine de Martres- France de la compagnie Lafarge.

- Ciment B : CEM I 52,5 R de l'usine Teil de Lafarge en France.

- Ciment C : CEM II/A 42,5 N de la cimenterie de Chlef (ECDE), Algérie.

Le sable est choisi selon la norme EN 196-1 [16], commercialisé en sacs plastiques de $1350 \pm 5 \mathrm{~g}$. L'eau de gâchage est une eau potable de robinet. La roche volcanique andésitique est de provenance la région de Tipaza, appartenant à la partie centrale du Nord Algérien, de réserve géologique d'environ 54 milliards $\mathrm{m}^{3}[9,12-$ 13]. Elle est composée principalement de Feldspath, Cristobalite, Quartz, Montmorillonite, Calcite et Dolomite (Figure 1), sa masse volumique apparente est de $2,30 \mathrm{~g} / \mathrm{cm} 3$. Les compositions chimiques et minéralogiques des différents constituants utilisés sont données au

Tableau 1. Compositions Chimiques et minéralogiques des matériaux utilisés

\begin{tabular}{|c|c|c|c|c|c|c|}
\hline \multicolumn{7}{|c|}{ Compositions Chimiques des matériaux utilisés } \\
\hline Matériaux & $\mathrm{SiO}_{2}$ & $\mathrm{Al}_{2} \mathrm{O}_{3}$ & $\mathrm{Fe}_{2} \mathrm{O}_{3}$ & $\mathrm{CaO}$ & MgO & $\mathrm{K} 2 \mathrm{O}$ \\
\hline CEMI52.5R & 22,84 & 2,70 & 1,84 & 67,41 & 0,81 & 0,23 \\
\hline Clinker & 21.50 & 5.18 & 3.76 & 66.36 & 0.95 & 0.66 \\
\hline Andésite & 57,25 & 14,97 & 5,96 & 8,95 & 1,97 & 1,24 \\
\hline Matériaux & $\mathrm{Na}_{2} \mathrm{O}$ & $\mathrm{TiO}_{2}$ & $\mathbf{P}_{2} \mathbf{O}_{5}$ & $\mathrm{SO}_{3}$ & $\mathbf{P F}$ & \\
\hline CEMI52.5R & 0,14 & - & - & 2,23 & 1,72 & \\
\hline Clinker & 0.11 & - & - & 0.78 & 0.48 & \\
\hline Andésite & 1,76 & 0,62 & 0,40 & 2,67 & 3,27 & \\
\hline \multicolumn{6}{|c|}{ Composition minéralogique des ciments utilisés } & \\
\hline Phases & $\mathrm{C}_{3} \mathrm{~S}$ & $\mathrm{C}_{2} \mathrm{~S}$ & $\mathbf{C}_{3} \mathrm{~A}$ & $\mathrm{C}_{4} \mathrm{AF}$ & Gypse & \\
\hline $\begin{array}{c}\text { CEM I } \\
52,5 \mathrm{R}\end{array}$ & 73,6 & 10,4 & 4,0 & 5,6 & $\sim 3$ & \\
\hline Clinker & 61,02 & 15,63 & 7,37 & 11,43 & $\sim 5$ & \\
\hline
\end{tabular}

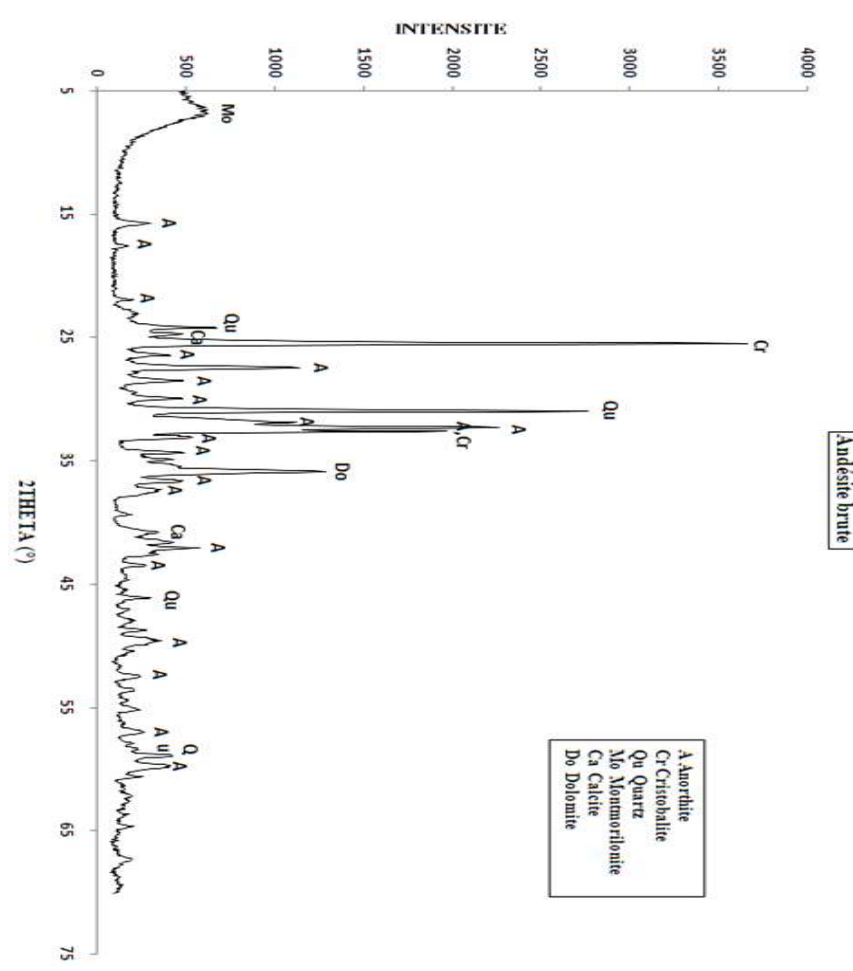

Fig. 1. Diffractogramme RX de l'andésite brute

\subsection{Méthodes d'essais}


Pour la caractérisation mécanique, l'étude expérimentale a été menée sur des éprouvettes de mortiers de 40x40x160 mm3, conformément à la norme EN 196-1 [16]. Les mortiers sont élaborés suivant les rapports massiques $(\mathrm{S} / \mathrm{C}=3$ et $\mathrm{E} / \mathrm{C}=0,5$ avec $\mathrm{C}$ : Ciment, $\mathrm{S}$ : Sable et E : Eau).

A chaque échéance d'âge, une série de trois (03) éprouvettes est soumise à l'essai de flexion et les six (06) demi-prismes obtenues sont soumis à l'essai de compression.

by the publisher.

\section{Résultats et discussions}

\subsection{Evolution des résistances mécaniques}

Les résultats des essais mécaniques obtenus pour les différentes éprouvettes des mortiers à base des trois ciments $\mathrm{A}, \mathrm{B}$ et $\mathrm{C}$ à ajout andésitique brut et calciné à différentes températures $\left(700^{\circ} \mathrm{C}, 800^{\circ} \mathrm{C}\right.$ et $\left.900^{\circ} \mathrm{C}\right)$ après $2,7,28$ et 90 jours de durcissement sont présentés dans le tableau 2.

Tableau 2. Résistances de mortiers à base des trois ciments A, $\mathrm{B}$ et $\mathrm{C}$ à ajout andésitique

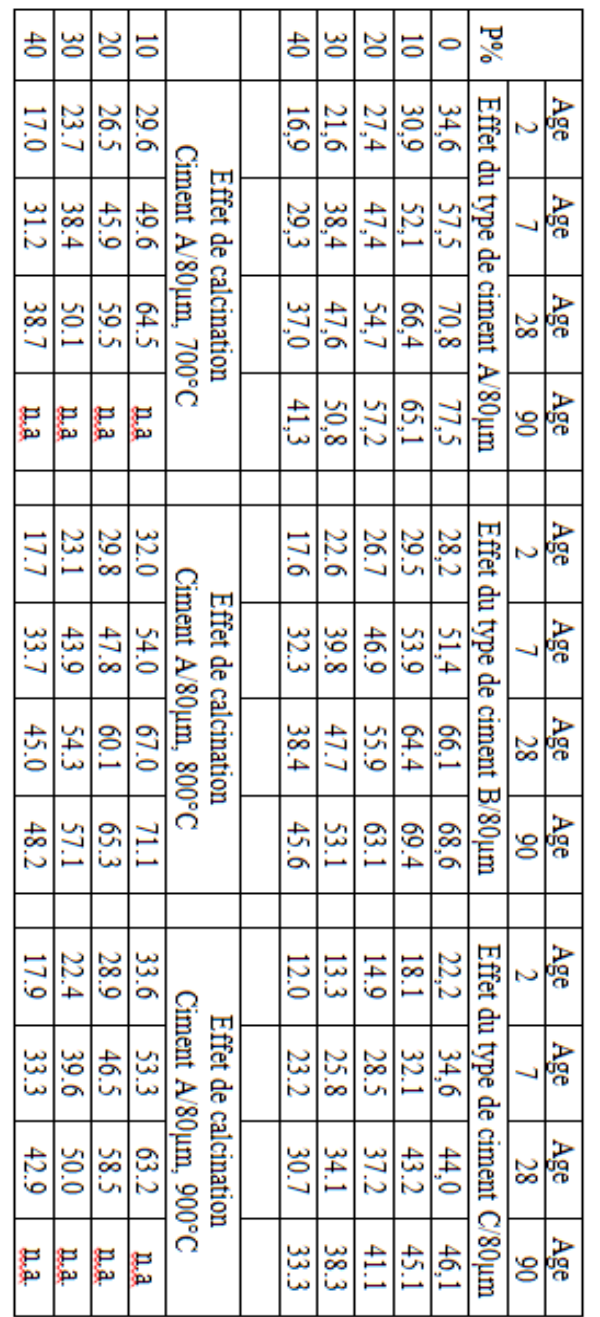

\subsection{Utilisation de l'andésite dans les ciments comme constituant principal}

Les travaux effectués par [14], ont montré que l'andésite a une activité pouzzolanique modérée et pourrait probablement être considérée comme une pouzzolane naturelle au sens de la norme EN 197-1 [15]. Le Tableau 3 présente quelques exemples de ciments qui pourraient être produits, selon les exigences mécaniques et la norme EN 197-1 [15] pour les ciments A, B et C utilisés dans cette étude.

Tableau 3. Exemples de ciments pouvant être produits en utilisant l'andésite, sur la base des exigences mécaniques et de composition de la norme EN 197-1 [15], et sur les travaux présentés dans [14]

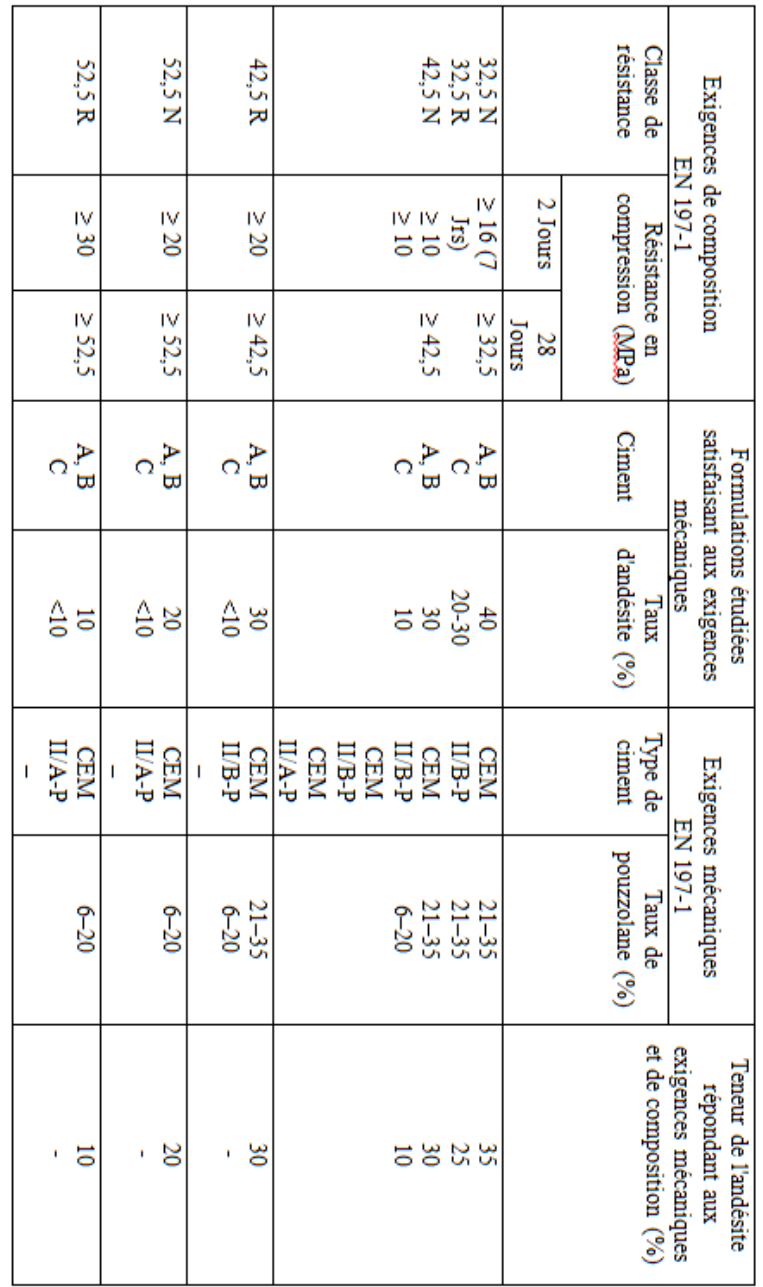

Nous pouvons voir que, selon le clinker choisi, toutes les classes de résistance pourraient être fabriquées et ce en allant jusqu'à 35\% d'andésite. Le présent travail ne permet de proposer que le ciment de type CEM II, mais il est possible de fabriquer des ciments de type CEM IV (par exemple, à base d'andésite et de cendres volantes) et CEM V (à base d'andésite et de laitier, d'andésite ou de laitier et de cendres volantes), à condition d'effectuer des tests supplémentaires. De toute évidence, des essais supplémentaires doivent être effectués comme, par exemple, le broyage simultané du clinker et de l'andésite, qui doit être effectué dans les mêmes conditions (même 
appareillage et même temps). En outre, le liant doit être optimisé, par exemple en ajustant la teneur en sulfate.

\subsection{L'andésite est-elle un matériau éco-efficace?}

L'andésite peut-elle être considérée comme un matériau éco-efficace lorsqu'elle est utilisée dans les matériaux cimentaires? A première vue, nous pourrions avancer que le remplacement du clinker ou du ciment par un ajout minéral est une bonne alternative pour la production de liants économiques et écologiques acceptables. En vue de répondre à cette question, un bilan complet du cycle de vie serait nécessaire, mais c'est audelà du cadre de la présente étude. Néanmoins, en vue de proposer des pistes de réflexion, nous présentons une analyse reposant sur un calcul simple de demande en énergie quand l'andésite est utilisée en tant qu'ajout de remplacement de ciment. Ce calcul est basé sur l'énergie consommée pour la production de ciment et d'andésite, qui comprend le procédé de fabrication (par exemple, le traitement thermique de la poudre crue du ciment, la calcination de l'andésite) et le broyage final des matériaux (clinker et andésite). L'énergie nécessaire (E, exprimée en $\mathrm{kWh} / \mathrm{t}$ de liant) est donnée par l'équation (1), en fonction de la composition du liant et de l'énergie nécessaire au procédé de fabrication et de broyage des matériaux (ciment et andésite).

$E(k W h / t)=C .\left(E_{p c}+E_{b c}\right)+A .\left(E_{p a}+E_{b a}\right)$

Le calcul de l'énergie de performance $E_{\text {perf. }}$ (en $k W$ $h / t / M P a)$ se fait en utilisant l'équation (2), en divisant l'énergie nécessaire $E$ par la résistance à la compression du mortier contenant le liant de l'étude. $E_{\text {perf., donne le }}$ coût de l'énergie de chaque $1 \mathrm{MPa}$ de résistance.

$E_{\text {perf. }}[(k W h / t) / M P a]=E / R_{c}$

$E$ : l'énergie de consommation (en $k W h$ ) pour la production d'une tonne de liant.

$C$ et $A$ : les proportions de ciment et d'andésite dans le liant respectivement.

$E_{p c}$ et $E_{b c}$ : les consommations d'énergie (en $k W h / t$ ) du procédé de fabrication et du broyage final du ciment, respectivement.

$E_{p a}$ et $E_{b a}$ : Les consommations d'énergie (en $\left.k W h / t\right)$ du procédé de calcination et du broyage final de l'andésite, respectivement.

$E_{\text {perf. }}$ La performance de consommation énergétique pour la production d'une tonne de liant, par rapport à la résistance en compression du mortier contenant le liant considéré.

$R_{c}$ : La résistance en compression du mortier de composition de $C+A$.

Trois cas ont été considérés :

1. L'andésite naturelle, n'ayant pas besoin de calcination mais devant être broyée (l'andésite a été considérée comme déjà dans un état granuleux, c'est à dire qu'aucune énergie n'a été prise en compte pour l'extraction de la roche).

2. L'andésite calcinée, qui doit être calcinée et broyée.
3. Les fines d'andésite naturelle provenant du procédé de broyage des agrégats de carrière. Dans ce cas, les fines ont été considérées comme des sous-produits, sans besoin de calcination ou de broyage supplémentaire.

Les données utilisées pour le calcul ont été prises de la littérature et sont reportées dans le tableau 4 [17-25].

Tableau 4. Données utilisées pour le calcul de la consommation d'énergie

\begin{tabular}{|c|c|c|c|c|}
\hline & \multicolumn{4}{|c|}{ Consommation d'énergie $(\mathrm{kWh} / \mathrm{t})$. } \\
\hline & $\begin{array}{c}\mathbf{C i} \\
\text { ment }\end{array}$ & ${ }_{\text {nce }}^{\text {Référe }}$ & Andésite & ${ }_{\text {nce }}^{\text {Référe }}$ \\
\hline $\begin{array}{r}\text { Procé } \\
\text { fabricatio } \\
E_{p c}=950= \\
\text { Broya } \\
E_{b c}=50\end{array}$ & $\begin{array}{l}\text { dé de } \\
n \\
950^{(a)} \\
\text { ge final }\end{array}$ & $\begin{array}{c}{[17,} \\
20-21,24] \\
\quad[19, \\
23]\end{array}$ & $\begin{array}{l}\text { Brute }^{(\mathrm{b})} \\
\text { ou Calcinée } \\
E_{p a}=0 \text { ou } \\
500^{(\mathrm{c})} \\
\quad \text { Fines de } \\
\text { broyage ou } \\
\text { Agrégats de } \\
\text { sol } E_{b a}=0 \text { ou } \\
75\end{array}$ & $22]^{[18,}$ \\
\hline
\end{tabular}

(a) Les valeurs d'énergie dans la littérature se situent entre 800 et $1200 \mathrm{kWh} / \mathrm{t}$ pour le procédé sec (les données initiales en GJ/t, avec 3,6 GJ = $1000 \mathrm{kWh}$ ).

(b) Sans tenir compte du procédé d'extraction de la carrière.

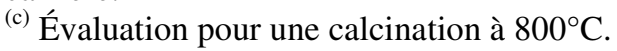

L'énergie totale requise pour produire du ciment a été évaluée dans la plage de $800-1200 \mathrm{kWh}$ par tonne de ciment, dont environ $50 \mathrm{kWh} / \mathrm{t}$ pour le broyage final du clinker. Dans le cas de d'andésite, l'énergie nécessaire pour le procédé de calcination a été évaluée à $500 \mathrm{kWh} / \mathrm{t}$ (température d'environ $800^{\circ} \mathrm{C}$ ). L'énergie de broyage d'andésite est admise comme étant plus élevée que celle du clinker, en raison de la présence de minéraux durs. Selon de nombreux auteurs [18, 22], plus de $50 \%$ d'énergie supplémentaire est nécessaire pour que la finesse de l'andésite atteigne celle du clinker. Les résultats du calcul sont donnés dans la Figure 2 pour des teneurs d'andésite jusqu'à $40 \%$.

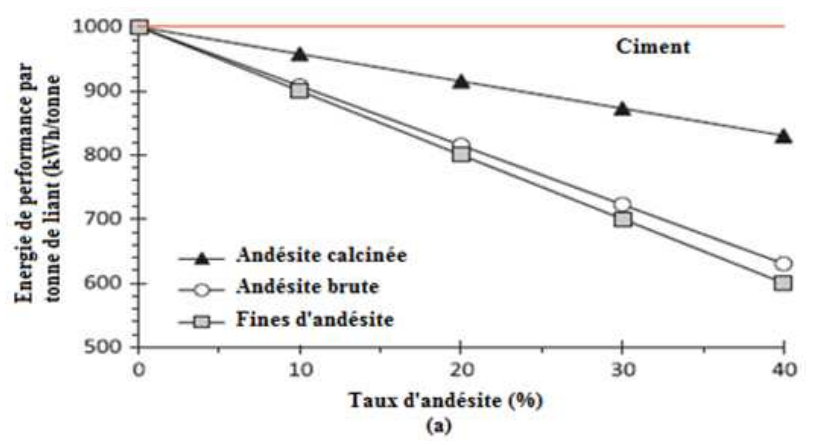




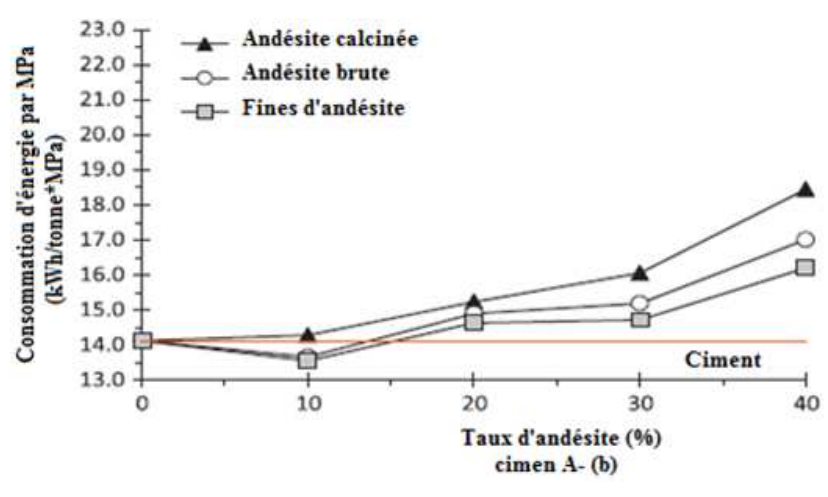

Fig. 2. Consommation énergétique de fabrication des ciments contenant de l'andésite.

(a) Besoin énergétique de liant ( $\mathrm{E}$, en $\mathrm{kWh} / \mathrm{t}$ de liant).

(b) Energie de performance (E performance, en $\mathrm{kWh} / \mathrm{t} / \mathrm{MPa}$ ) pour le ciment à 28 jours.

\subsection{Besoin énergétique du liant}

L'examen de l'énergie nécessaire pour une tonne de liant montre que la consommation d'énergie par le ciment d'andésite a toujours été inférieure à celle de la référence (ciment sans ajout) (Figure 2 (a)), bien que le broyage de l'andésite exige plus d'énergie que celui du clinker. L'andésite reste moins consommatrice d'énergie, en raison de la demande énergétique plus faible du procédé de fabrication (sans prendre en compte l'extraction de la roche). Il est évident que l'andésite calcinée augmente la demande en énergie d'une valeur qui se situe entre celle provoquée par l'andésite naturelle et le témoin (un liant de $100 \%$ ciment). Dans le cas des fines d'andésite naturelle, sous-produit provenant du procédé de broyage d'agrégats, un faible gain d'énergie a été enregistré comparativement à l'andésite naturelle.

\subsection{Energie de performance}

Lorsque la consommation d'énergie est considérée par rapport à la performance des mortiers (par exemple par rapport à la résistance en compression à 28 jours), les conclusions sont très différentes de celles tirées de la partie du «besoin énergétique » car, selon le ciment utilisé et le taux d'andésite, des points se trouvent sous ou au-dessus de la courbe de référence (ciment sans ajout). Dans le cas du ciment A (Figure 2 (b)), la plupart des liants d'andésite exigent plus d'énergie comparativement au ciment ordinaire, en particulier pour des taux de remplacement d'andésite élevés. Cela signifie qu'une résistance d'un $\mathrm{MPa}$ exige plus d'énergie, en raison de l'activité pouzzolanique modérée de l'andésite. L'andésite calcinée a fait le cas le plus défavorable, avec une demande d'énergie du ciment supérieure à celle du ciment témoin et les ciments à andésite brute. L'augmentation de la résistance du ciment due à la calcination de l'andésite n'a pas été suffisamment élevée pour compenser la hausse de consommation énergétique de fabrication. Parmi les formulations testées du ciment A, seul le liant avec 10\% d'andésite a conduit à de meilleurs résultats par rapport à la référence de point de vue consommation énergétiqueperformance des mortiers. Les besoins énergétiques pour les liants à 20 et $30 \%$ d'andésite ont été légèrement supérieurs mais restent assez proches de la référence. Comme nous l'avons remarqué, les fines d'andésite naturelle provenant du procédé de broyage d'agrégats ont été le meilleur choix pour un taux de substitution de $30 \%$ d'andésite. Il est à noter que les résultats de performance pourraient être améliorés si l'efficacité de l'andésite était améliorée, par exemple en choisissant un couple de ciment/andésite compatible. La Figure 2 (c) présente une comparaison de l'énergie de la performance pour des liants fabriqués composé d'andésite naturelle et de ciments A, B ou C. Nous pouvons constater que le type de ciment a une grande influence sur les résultats. Les consommations d'énergie (par rapport à la résistance en compression) des ciments d'andésite ont été inférieures ou égales à celles du ciment seul pour les différentes teneurs d'andésite : 10, 30 et plus de $40 \%$ pour les ciments $\mathrm{A}, \mathrm{B}$ et $\mathrm{C}$ respectivement. Ces résultats étaient prévisibles puisque les performances d'andésite ont été très différentes d'un ciment à autre. Cela signifie que, d'un point de vue rendement énergétique, il est préférable de n'utiliser l'andésite qu'avec des ciments ayant une efficacité modérée, comme le ciment $\mathrm{C}$ dans la présente étude. Pour ce ciment, chaque $1 \mathrm{MPa}$ vaut moins de 1 $\mathrm{kWh}$ par tonne de liant, quelle que soit la teneur de l'andésite. Ce résultat peut être intéressant pour des pays en développement, où les ciments ont généralement de faibles performances. C'est le cas ici avec le ciment C, qui est de provenance algérienne. En résumé, nous pouvons dire que l'énergie nécessaire pour produire du liant (Figure 2 (a)) est systématiquement la plus faible lorsque l'andésite est utilisée, mais de point de vue rendement énergétique (pour atteindre un niveau donné de résistance, Figure 2 (b) et (c)), l'andésite peut être qualifiée comme écologiquement acceptable seulement dans certains cas. Suivant le point de vue adopté, la conclusion peut être très différente. Il est probable que l'application d'une approche similaire pour d'autres paramètres environnementaux, tels que la production de $\mathrm{CO} 2$, conduirait à des conclusions similaires, car les émissions de $\mathrm{CO} 2$ suivent la même tendance : une forte émission de $\mathrm{CO} 2$ pour la production de clinker contre une plus faible émission de $\mathrm{CO} 2$ pour l'andésite.

\section{Conclusions}

Dans la présente étude, nous avons étudié l'impact environnemental de la valorisation de l'andésite en substitution du ciment dans la matrice cimentaire. L'analyse de l'influence de cette addition, à l'état brut et calcinée à $800^{\circ} \mathrm{C}$, sur l'environnement a permis de tirer les conclusions suivantes :

- Les principaux constituants de l'andésite utilisée sont : Feldspath, Cristobalite, Quartz, Montmorilonite, Calcite et Dolomite et la somme de ces oxydes majeurs est de 78,18\%. Selon la norme ASTM C 618, cette andésite peut être considérée comme matériau pouzzolanique.

- L'incorporation progressive de la roche andésitique entraîne une légère diminution de résistances 
mécaniques. Toutefois, à l'âge de 28 et 90 jours, les mortiers contenant jusqu'à $10 \%$ d'andésite peuvent se comparer avec le produit témoin.

- Globalement l'impact de l'andésite sur les matrices à long terme semble positif.

- Le remplacement du ciment par l'andésite induit systématiquement une diminution de l'énergie nécessaire pour produire une tonne de liant, en raison de la demande d'énergie de broyage moins faible par rapport au traitement thermique du clinker.

- Par rapport à la performance du mortier, le coût énergétique du ciment d'andésite (en $\mathrm{kWh}$ par $\mathrm{MPa}$ de résistance à la compression) est inférieur à celle du ciment ordinaire pour les faibles taux de remplacement et les ciments présentant une efficacité modérée.

\section{Références}

1. D. Bondar, C. J. Lynsdale, N. B. Milestone, N. Hassan, Geopolymer Cement from Alkali-Activated Natural Pozzolans : Effect of Addition of Minerals, 2nd International Conference On Sustainable Construction Materials and Technologies, Università Politecnica delle Marche, Ancona, Italia, 28-30 June, (2010)

2. Y. Tatsumi et T. Akahashi, Operation of subduction factory and production of andesite, (JAMSTEC), Vol.101 (1), P.145-153, (2006).

3. P. M. Black, Andesites as resources for roading and concrete industries, North Island of New Zealand, Department of Geology, University of Auckland, Auckland 1020, P. 328-332, (2009).

4. Takanori, Y. Kawabata, H. Hamada, Y. Sagawa, Alkali-Silica Reactivity of Andesite in $\mathrm{NaCl}$ Saturated Solution, International Conference on Durability of Concrete Structures, China, P. 1-8, (2008).

5. U. Chinje melo, N. Billong, Activité pouzzolanique des déchets de briques et tuiles cuites. Laboratoire des Matériaux et du Contrôle de Qualité, (AJST), vol. $5 \mathrm{~N}^{\circ} 1$, P. 92-100, (2004).

6. Y. Kawabata, 2007. Petrological study on evaluating of alkali-silica reactivity and expansion analysis of andesite, Doboku Gakkai Ronbunshuu, E (63), P. 689-703, (2007).

7. M. Saidi, 2010. Development and Structural Study of Cements Containing Additions of Industrial Waste. European Journal of Scientific Research Vol.40 (2), P. 297-306 (2010).

8. S. Martínez-Ramírez, M.T. Blanco Varela, I. Ereña, M. Gener, Pozzolanic reactivity of zeolitic rocks from two different Cuban deposits: Characterization of reaction products, Applied Clay Science vol. 32, P. 40-52, (2006).

9. F. Ait Hamou, Etude pétrographique et géochimique du volcanisme d'âge miocène de la région de Hadjout (Ouest Algérois), Thèse de magister en géologie, (1987).

10. G. G. Gvazava, O. I. Chokhonelidze, G. Sh. Kurtanidze, V. I. Kharazishvili, N. D. Natbeladze,
Use of Bakury Andesite in the production of darkgreen bottles, Glass and Ceramics, vol. $47 \mathrm{~N}^{\circ} 10, \mathrm{P}$. 371-374, (1990).

11. A. DAA, et H.M. El-Kaliouby Utilisation d'andésite dans la transformation des organismes électrocéramiques, Les progrès de la céramique appliquée, vol.106 (3), P. 113-119, (2007).

12. D. Belhai Evolution tectonique de la zone ouestAlgéroise, Approche Stratigraphique et structurale, Thèse de Doctorat d'Etat, USTHB, Algérie, (1996).

13. H. Benali, Les minéralisations associées aux roches magmatiques tertiaires du nord de l'Algérie, Thèse de Doctorat d'Etat, U.S.T.H.B, Algérie, (2003).

14. M. Hamidi Elaboration d'un éco-ciment composé à base d'un ajout andésitique brut et activé, Thèse Docteur ès Sciences, USTO, Oran Algérie, (2014).

15. Norme AFNOR. NF EN 197-1, Cement. Composition, specifications and conformity criteria for common cements, (2011).

16. Norme EN 196-1 Méthodes d'essais des ciments Partie 1 : Détermination des résistances mécaniques, (2005).

17. IZ. Bribián et al., Life cycle assessment of building materials: Comparative analysis of energy and environmental impacts and evaluation of the ecoefficiency improvement potential. Build Environ, 46(5), 1133-40, (2011).

18. JN. De La Vergne The hard rock miner's handbook. $3^{\text {rd }}$ Ed. McIntosh Engineering: North Bay, (2003).

19. C. Hosten, B. Fidan An industrial comparative study of cement clinker grinding systems regarding the specific energy consumption and cement properties. Powder Technol. 221:183-8, (2012).

20. NA. Madlool et al., A critical review on energy use and savings in the cement industries. Renew Sustainable Energy Rev 15(4): 2042-60, (2011).

21. JA. Moya et al., Pardo N, Mercier A. The potential for improvements in energy efficiency and $\mathrm{CO} 2$ emissions in the EU27 cement industry and the relationship with the capital budgeting decision criteria. J Clean Prod 19(11), 1207-15, (2011).

22. RH. Snow et al., Size reduction and Size Enlargement. In Perry RH, Green DW, editors. Perry's Chemical Engineers, Handbook. 7th Edition. McGraw-Hill, (1997).

23. D. Touil, S. Belaadi, C. Frances Energy efficiency of cement finishes grinding in a dry batch ball mill. Cement Concrete Research, 36(3) : 416-21, (2006).

24. World Business Council for Sustainable Development (WBCSD). Cement Sustainability Initiative (CSI). Global Cement Database on $\mathrm{CO} 2$ and Energy Information, $<$ http://wbcsdcement.org/index.php?Itemid=74>.

25. E. Worrell et al., Potentials for energy efficiency improvement in the US cement industry. Energy 25(12) :1189-214, (2000). 